\title{
Training of Rhesus Macaques to Complex Cognitive Tasks
}

\author{
I. V. Bondar, ${ }^{1}$ L. N. Vasileva, ${ }^{1}$ L. V. Tereshchenko, ${ }^{2}$ \\ A. V. Buynevich, ${ }^{2}$ and A. V. Latanov ${ }^{2}$
}

UDC 612.833 .81

\begin{abstract}
Translated from Zhurnal Vysshei Nervnoi Deyatel'nosti imeni I. P. Pavlova, Vol. 68, No. 4, pp. 459-476, July-August, 2018. Original article submitted January 16, 2018. Accepted May 14, 2018.

The closeness of the evolutionary origin, physiology, and structure of the brain with humans makes lower primates the preferred experimental animals for biomedical research. Many brain diseases induce degradation of cognitive capacities. Various aspects of impairments to cognitive functions are studied in model experiments in monkeys. In the present article we discuss the theoretical grounds for learning complex tasks by primates and present results from training animals using three different behavioral tasks: an oculomotor task, a visual objects categorization task, and a task for assessment of the retention of motor function on extraction of a bait. The results indicate that these tests can be used simultaneously in one animal. This approach leads to optimization of models for studies of cognitive impairments associated with brain diseases.
\end{abstract}

Keywords: lower primates, model objects, impairments to cognitive functions, learning in monkeys, categorization, eye movements, learning.

The current understanding of the etiology of neurological and mental diseases is based on the combined actions of genetic and environmental factors. The view that the increase in the number of cases of neurodegenerative diseases is linked with increases in longevity is widespread. Understanding of the pathological processes underlying nervous system diseases requires animal experiments. Modeling of diseases in animals must ensure similar etiology, demonstrate a realistic picture of the course of the pathological processes, and have sufficient predictive power [Camus et al., 2015].

Monkeys are the preferred experimental animals for biomedical research because they have the greatest anatomical, physiological, and biochemical similarity with humans [Phillips et al., 2014]. Humans and primates share a common evolutionary origin, complex cognitive functions, and developed social organization; they have similar reproductive cycles and similarity in the organization of the brain.

\footnotetext{
${ }^{1}$ Institute of Higher Nervous Activity and Neurophysiology, Russian Academy of Sciences, Moscow, Russia; e-mail: bondar@ihna.ru.

${ }^{2}$ Department of Higher Nervous Activity, Faculty of Biology, Lomonosov Moscow State University, Moscow, Russia; e-mail: latanov@neurobiology.ru.
}

Rhesus macaques (members of the Cercopithecoidea superfamily, or lower narrow-nosed monkeys) are used for drug testing, vaccine development, and research into the mechanisms of various diseases [Roelfsema and Treue, 2014]. Data from various experiments, including brain mapping, have shown the fundamental similarity of the neural networks of primates and humans, such that they can be compared with each other. Thus, similarity has been demonstrated between the neural mechanisms of working memory [Goldman-Rakic, 1995], decision-taking, and assessment of information relating to reinforcement and the functioning of mirror neurons [Rizzolatti et al., 1996], as well as the processing of information relating to faces and their expressions [Tsao et al., 2008]. Social behavior in macaques provides rich material for modeling mental disorders, as it becomes possible to influence interactions in a group, maternal behavior, and the exchange of information between individuals [Camus et al., 2015]. Lower primates are used for studies of the long-term sequelae of ionizing radiation [Hanbury et al., 2016]. Investigations of cognitive impairments in experiments on monkeys provide for studies of the pathophysiological mechanisms, severity, and prognosis of the course of nervous system diseases and for treatment methods to be developed. 
Macaques yield entirely adequate models of human neurological diseases such as Parkinson's disease, which is linked with dysfunction of the dopaminergic system. Administration of the neurotoxin 1-methyl-4-phenyl-1,2,3,6-tetrahydropyridine (MPTP) leads to the death of dopaminergic cells in the substantia nigra and, as a result, Parkinson's disease [Chiueh et al., 1984; Langston et al., 1984]. This substance is used to model parkinsonism in animals with the aim of developing effective treatments [Jenner, 2009; Porras and Bezard, 2012; Yan et al., 2014]. MPTP induces parkinsonism-like syndrome in macaques, accompanied by impairments to visuomotor coordination [Latanov et al., 1997; Tereshchenko et al., 2002; Tereshchenko et al., 2015; DeLong, 1990]. Degradation of the performance of a spatial working memory task is seen, which is typical of patients with this disease. Electrophysiological methods have demonstrated the features of changes in neuron activity in the basal ganglia, which may underlie the pathological changes in Parkinson's disease [Wichman and DeLong, 2003].

Use of scopolamine - a nonselective muscarinic cholinoreceptor agonist - to some extent provides a model of Alzheimer's disease in lower primates. Treatment of macaques with this substance increases the proportion of errors in a delayed example-based choice task. Alzheimer's disease can also be studied and modeled by extirpation of certain brain areas in macaques. These data made it possible to test drugs for their ability to ameliorate the symptoms of Alzheimer's disease [Capitanio and Emborg, 2008].

Observations of the behavior of animals in natural social groups allowed specific behavioral markers of depressive states to be identified, producing primate models of depression [Camus et al., 2015]. Monkeys can be used for research into prenatal and postnatal stress, leading on to studies of the effects of stressors on cognitive functions [Capitanio and Emborg, 2008].

Macaques are used for studies of neurological problems apparent in acquired immunodeficiency syndrome (AIDS). Symptoms of dementia appear at the late stages of this disease. Experiments on macaques have shown that simian immunodeficiency virus enters the nervous system with macrophages and monocytes [Williams et al., 2001, cited by Capitanio and Emborg, 2008]. It is clear that keeping peripheral virus titers low is extremely important for decreasing the risk of damage to the central nervous system by immunodeficiency virus. Studies of cognitive functions in sick animals - spatial working memory, long-term memory, and motor dysfunctions - play a particular role.

Studies of cognitive functions presume the use of standard behavioral tests providing data which can be compared between humans and animals. The Cambridge Neuropsychological Test Automated battery (CANTAB) was specially developed to test various aspects of the operation of the brain in primates. It provides for assessment of, among others, visuospatial learning, spatial working memory, stimulus discrimination ability, and motor coordination. Visuospatial learning requires the involvement of working memory. During task execution, the animal is presented with a prompt signaling the future position of the target stimulus. Responses are regarded as correct when the animal points to the stimulus located in the position indicated by the prompt [Nagahara et al., 2010]. On testing of spatial working memory, the animal is presented with a set of stimuli on a touchscreen. Correct performance of trials requires sequential touching of each stimulus on the screen without repetition. Such a trial is regarded as successful and is reinforced [Taffe and Taffe, 2011]. Spatial working memory requires normal functioning of the frontal areas of the brain. Correct execution of the two-stimulus discrimination task requires the animal to learn a rule: only the figure with the closed outline is reinforced, while background thin lines in different orientations must be ignored [Moss et al., 1997]. Testing with different time intervals (from $1 \mathrm{~h}$ to days) allows retention of information in long-term memory to be evaluated. In the simplest case, motor coordination is assessed by extraction of small objects from a special device, which requires coordinated operation of the two hands [Nagahara et al., 2010]. In delayed response tasks, the subject is presented with some stimulus (visual or auditory) and, after some seconds, the ability to retain information in memory is tested, the test using presentation of the same stimulus. This type of task establishes the role of the hippocampus and the prefrontal and medial temporal cortex in short-term memory, control, and attention processes. These are extremely popular in primates and are used in parallel with recording of neuron activity or extirpation of particular brain areas with the aim of identifying their roles in supporting particular functions [Lind et al., 2015; Rodriguez and Paule, 2009].

Summarizing these points, we note that primates are the most preferred system for studies of impairments of cognitive processes in models of brain diseases of different etiologies. Successful testing of nervous system functionality can be achieved by creating standard training methods. An important element for developing such methodological approaches is assessment of the animal's dynamics of acquisition of particular skills. The aim of the present work was to assess the dynamics of the acquisition of operant reflexes in visually induced saccade execution tasks and image categorization tasks involving the oculomotor system, and performance of food reinforcement extraction tasks.

Methods. Experiments were performed on four rhesus macaques (Macaca mulatta, Zimmermann, 1780). All animals were adult males weighing $5-8 \mathrm{~kg}$ (VL was 5 years old, GR was 5 years old, DK was 23 years old, and BL was 15 years old). All animal manipulations were carried out in compliance with the requirements of the Directives of the European Parliament and the Council of the European Union (2010/63/EU) on the use of animals for experimental investigations.

All monkeys were initially acquired from the Institute of Medical Primatology (Krasnodar District), where the ani- 
mals were kept in open corrals in large groups. Animals DK and BL were transported to the animal house of the Faculty of Biology, Lomonosov Moscow State University in the 1990s after being kept at the Institute for Medical Biological Problems, Russian Academy of Sciences. The younger animals (GR and VL) were transported to the Scientific Teaching Center, Biological Faculty for the Rehabilitation of Wild Animals (Lomonosov Moscow State University) at age 4 years and were later transported to the animal house of the Institute of Higher Nervous Activity and Neurophysiology, Russian Academy of Sciences. Animals were kept in individual cages of size $45 \times 70 \times 75 \mathrm{~cm}$ or $70 \times 80 \times 80 \mathrm{~cm}$. Alternation of the light and dark parts of the day in the animal house location were generally consistent with natural seasonal variations; additional artificial illumination ensured a 12:12 h rhythm in the autumn-winter period. Animal experiments were performed mainly in the first half of the day.

When studying the long-term effects of different treatments on higher nervous activity in primates, investigators generally encounter the need to study a wide range of behavioral parameters. In the practice of science, it is not always possible to include all the behavioral measures required for the experiment in the program of a single experiment. When an animal is working in parallel in several experiments, investigators come up against the problem that the motivation developed in the animals does not allow the required volume of data to be obtained in each experiment. The animals' work capacity in this situation can be increased if the food and drink needs of the animals are both involved. These needs hardly interact with each other: drinking liquid provides barely any satiety, while food, if juicy fruits are excluded from the diet during the experiment, hardly satisfies thirst. This allows independent formation and utilization of the food and drink motivations in two experiments in parallel using the corresponding motivation in one independently of the success of the animal's work in another experiment.

The drink motivation can operate in an automated experiment in which a controllable pump can be used to provide dosed delivery of small volumes of liquid $(0.1-0.3 \mathrm{ml})$ as drink reinforcement. Food reinforcement can be used in both automated experiments in which chunks of food are released with an automated doser, and in experiments addressing the components of the animal's natural behavior, in which successful performance by the monkey consists of the monkey reaching for chunks of food with the hand.

With the experiment set up in this way, monitoring of the monkey's condition and weight acquires particular importance in terms of avoiding dehydration and exhaustion of the animals in the unavoidable need to maintain a defined level of food and drink deprivation.

This article describes experimental methods used for studies of primate behavior which can be used simultaneously in long-term experiments on behavior in monkeys.

Oculomotor task. The experiment used an automated experimental programmable system. The minimal config- uration of the system included the following components: a primatology chair; a computer-controlled monitor screen for presentation of visual objects; manipulators for the animals'; motor responses; a pump for delivering drink reinforcement; a commutator block controlling presentation of the visual stimuli, manipulators, and pump; a computer with software controlling the experiment and real-time analysis of the indicators of the animal's responses. Experiments were run in an automatic regime. The effects of side factors were excluded by placing the animal on a primate chair in a light- and sound-proofed working room.

The task was to develop a chained operant reflex to visual stimuli in the monkeys. This behavioral model is used in studies of the functions of the oculomotor system.

At the final stage of development of the operant reflex, the animal had to respond correctly to a defined sequence of events in each trial. The visual stimuli were presented on a monitor screen of size $40^{\circ} \times 30^{\circ}$. Each trial started with the appearance of a white square (the central stimulus, CS) at the center of the screen. As soon as the animal fixed its gaze on the CS, another white square of the same size (the peripheral stimulus, $\mathrm{PrS}$ ) appeared in another location on the monitor and the CS disappeared. The animal transferred its gaze to the new stimulus, after which the PrS was replaced by a gray square (the trigger stimulus, TrS). In response to the change in brightness of the TrS, the monkey carried out the operant reaction (OR) - pulling a lever - after which a drink reinforcement was received. Stimulus size decreased as the animal's skill improved. The length of the sides of the squares initially appearing on the screen $(\mathrm{CS}$, $\operatorname{TrS}, \operatorname{PrS}$ ) was $21^{\circ}$, decreasing to $1.7^{\circ}$ by the final stage of training. Training of the animal to this complex reflex was performed stepwise.

Monkeys can be trained to produce differential responses with the right and left hands for the corresponding visual tasks. For example, when the TrS appears in the right-hand side of the field of vision, the monkey performs the correct OR using the right hand, movement of the left not being reinforced, and vice versa. This experimental scheme allows functional asymmetry of hand movements and visuomotor interactions between the eyes and hands to be studied [Tereshchenko et al., 2012].

At the first stage, the monkey was acclimated to the apparatus and received reinforcement within it. The animal was regularly placed in the primatology chair and rewarded with drink reinforcement. This stage of training could be regarded as complete when the animal spontaneously went to the chair and was familiar with receiving the reinforcement.

At the second stage, an association between the OR and receipt of the reinforcement was developed. Reinforcement was presented only after the animal had performed a lever movement of defined amplitude. The animal could only receive repeat reinforcement after returning the lever to its initial position. Monkeys demonstrated marked exploratory behavior and generally rapidly started to complete the re- 


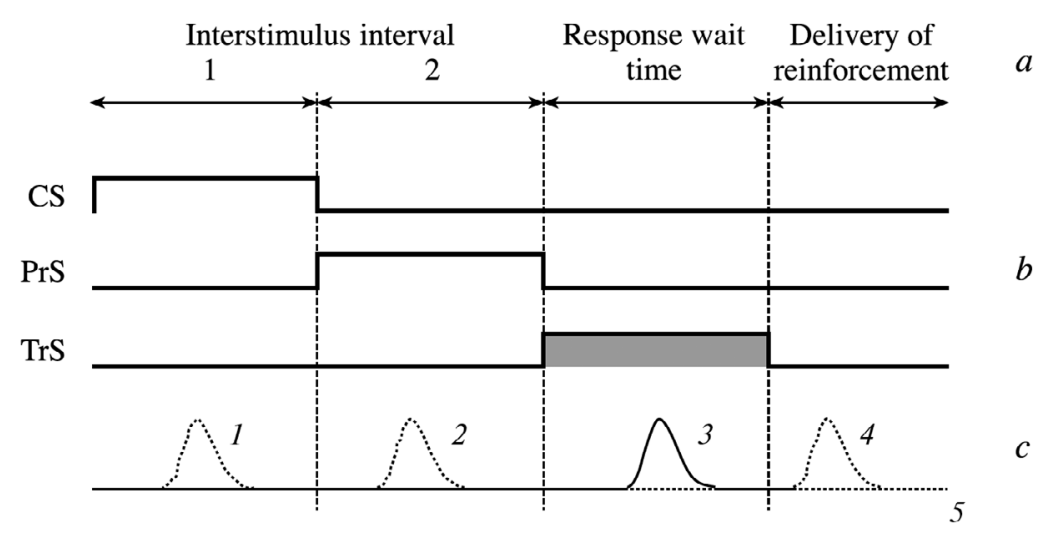

Fig. 1. Sequence of events in one operant task performance trial. CS - central stimulus; $\operatorname{PrS}$ - peripheral stimulus; TrS - trigger stimulus. a) Time interval for assessment of significant behavioral events within trial; $b$ ) time diagrams showing switching on and off of the visual stimuli; $c$ ) animal's reactions: 1-2) predictive reactions (anticipation); 3) correct responses; 4) late reactions.

quired movements, especially if the lever was comfortably positioned in front of the animal's hands.

The next stage consisted of developing a conditioned association between the $\mathrm{TrS}$ and the OR. A TrS was presented on the monitor screen, for example a stimulus consisting of a dim square. Training started with a large stimulus. Reinforcement was delivered only on performance of the OR during presentation of the TrS. The TrS was turned off for some period of time as the reinforcement was delivered. OR performed in the absence of the $\mathrm{TrS}$ were not reinforced. In the darkened chamber, switching on and off of the stimulus induced changes in illumination and, as a result, attracted attention. After some period of time, the animal established an association between the presence/absence of the stimulus and receipt/nonreceipt of reinforcement after execution of the OR. The proportion of correct responses increased to essentially $100 \%$ in trained monkeys. Stimulus size then decreased gradually until it reached the required size. Starting from this stage of training, pseudorandom changes in interstimulus intervals - the time between stimulus presentations - at this stage, the $\operatorname{TrS}$ - were introduced. Interstimulus interval durations had to vary in a pseudorandom manner between the minimum and maximum values. This means of stimulus presentation prevented formation of a reflex to time.

At the fourth stage, a CS was added before the TrS, in this case a white square in the center of the monitor. The presentation time changed in a pseudorandom way within a specified range. On execution of the OR, all stimuli were turned off and, after a variable time interval, the next sequence of stimuli was presented. Intersignal OR to bright CS were not reinforced. If the OR to a bright stimulus did not occur, then the trigger stimulus was delivered. OR within a fixed waiting period were regarded as correct and were reinforced. The absence of an OR during the waiting period was taken as a "refusal." Introduction of a fixed waiting time for correct OR was needed to exclude delayed motor responses from the number of reinforced responses, as these motor responses are not an appropriate reaction to the $\mathrm{TrS}$.
As the animal's learning progressed, the waiting time decreased. An important characteristic of OR in response to $\mathrm{TrS}$ was their latent period (LP) - the time interval between presentation of the trigger stimulus and the start of execution of the motor reaction, i.e., the time taken to prepare to perform the required movement.

At the final (fifth) stage, presentation of the PrS was added. The CS was turned off at the end of its presentation time and a bright $\operatorname{PrS}$ was presented in another position. The PrS presentation time was the same as the CS presentation time and was determined pseudorandomly within a specified time range. OR during presentation of the PrS were also assigned to interstimulus reactions and were not reinforced. The TrS was presented at the same site as the PrS, and when an OR was performed the reinforcement was delivered.

Several subtypes of trials were discriminated depending on the temporal and spatial relationships between the central and peripheral stimuli. In the simplest case, only one central stimulus appeared on the screen and changes in its intensity served as a signal to carry out the reaction (The "fix" condition). In other cases, trials could be distinguished by the time of appearance of the peripheral stimulus: "with a delay" (scheme 1, S1 for brevity), "without a delay" (S2), and "with overlap" (S3). In trials with S1, the peripheral stimulus appeared on the screen after the central stimulus was turned off. The interval between disappearance of Condition S2 was characterized by the simultaneous appearance of the CS and the PrS. Trials with condition S3 differed from all others in that the PrS appeared on the screen some period of time after appearance of the CS, the CS remaining in the animal's field of vision for a period of time. Use of different schemes of presentation of the CS and PrS could influence switching of the processing of information on the CS and the Prs in the oculomotor system. During a single experimental day, the animal was presented with sequences of stimuli (CS, PrS, and TrS) until it displayed the correct OR. All OR performed by the monkeys in the experiment could be discriminated into three types: 
interstimulus OR - OR performed on presentation of bright CS and PrS ( 1 and 2 in Fig. 1), correct OR - OR to TrS (3 in Fig. 1), and "refusals" - OR at the end of the response waiting period or the absence of OR (4 and 5 in Fig. 1), reinforcement not being delivered.

Important parameters characterizing learning and work by the monkeys in the experiment were: the proportion of correct reactions (CR) and the proportion of refusals (Ref):

$\mathrm{CR}=($ number of correct $\mathrm{OR}) /$

(number of correct OR + number of interstimulus OR);

Ref $=$ (number of refusals $) /$

(total number of trials presented).

The parameters CR and Ref can be determined for each experimental day. Learning at stages 4 and 5 can be regarded as complete when CR is greater than $80-90 \%$ for some period of time (5-6 experimental days).

The fastest learning in monkeys requires a high level of motivation to be maintained in the animals. Use of a drink reinforcement requires reduction of the content of liquid in the animals' diet by replacing juicy food with drier food, so that the monkeys obtained as much of their liquid as possible via the experiment. The animals were fed after each experiment. The animals' physiological state was monitored after each experiment by regular weighing.

As learning proceeded, the monkeys received decreasing volumes of reinforcement on performance of a correct OR. At the final stage of training, the monkeys worked stably with receipt of $0.3-0.5 \mathrm{ml}$ of liquid per trial. In these working conditions, animals weighing $5-8 \mathrm{~kg}$ performed 800-1500 correct OR within a single experimental session.

The complete learning time for this conditioned operant reflex using this method is 4-5 months. In some monkeys, the operant reflex was acquired within three months.

Image categorization task. Experiments to train monkeys to categorizing images presented on the monitor screen were performed using one animal.

Presentation of stimuli on the monitor screen and delivery of reinforcement were run automatically using specialized software. We are aware of two noncommercial programs written in Matlab which provide for controlling task performance by experimental animals and run the course of the whole experiment. One of these - MonkeyLogic - is a system developed in Freedman's laboratory [Asaad et al., 2013], traditionally studying cognitive processes in monkeys. An undoubted advantage of this system is its ability to control all events to an accuracy of $1 \mathrm{msec}$. It provides for presentation of different images, sounds, and video clips to a subject. We used this system in experiments involving training monkeys to a categorization task. Two PCI-6221 data input-output cards (National Instruments, USA) provided independent digitization of signals from the joystick and the eye movements recorder, allowing all aspects of the animal's behavior to be followed. Depending on the correctness of task performance, this apparatus allows reinforcement delivery to be controlled.

The other system we are aware of is Psychophysics Toolbox [Brainard, 1997; Kleiner et al., 2007; Pelli, 1997]. In contrast to the previous system, this was developed for programming psychophysical experiments on humans, though it is also actively used for behavioral studies in animals [McMahon et al., 2015]. In our view, Psychophysics Toolbox has more diverse instrumentation for creating visual and auditory stimuli. Apart from presenting images and videos on screen, the system also allows moving stimuli to be created, with changes in their sizes if needed for the behavioral task. This system may be a worthy alternative to the MonkeyLogic system.

At the final stage of training to the categorization task, the animal had to have the skill of discriminating multiple images from two categories. The test verifies, on the one hand, the animal's ability to maintain information on concrete images for long periods and, on the other, to relate new stimuli to learned category rules.

In each trial, the animal is presented with a single image and must respond to each image with a motor response corresponding to the defined category. In our case, the monkey learned to categorize images from two categories - faces and butterflies. The monkey manipulated a joystick and had to respond to a butterfly by moving the joystick towards itself and to a face by moving it away. The monkey was trained to the task in several stages.

At the first stage, the animal was familiarized with the experimental apparatus and joystick, reinforcing when the animal was in the primatology chair and for any movement of the joystick.

At the second stage, images started to be presented on the monitor screen. It was important to acquire a correct operant reaction, so the screen showed colored squares rather than images. A blue square appeared at the bottom of the screen, and the monkey had to respond to this by moving the joystick towards itself as quickly as possible.

At the next stage, a yellow square was added to the task, presented at the top of the screen, and the joystick had to be moved away in order to receive the reward. The blue and yellow squares were now presented with different frequencies in a pseudorandom manner. The conditions for receiving the reinforcement on presentation of the blue square were not altered.

At the fourth stage, the squares were presented at the center of the screen and the required responses remained as previously. The animal could now orient itself solely in terms of the color of the square.

Transfer to the fifth stage involved addition of the images of a single face and a single butterfly to the task, presented on a yellow or blue background, allowing the association between a defined movement and a color to be transferred to specific images. As soon as the level of correct re- 


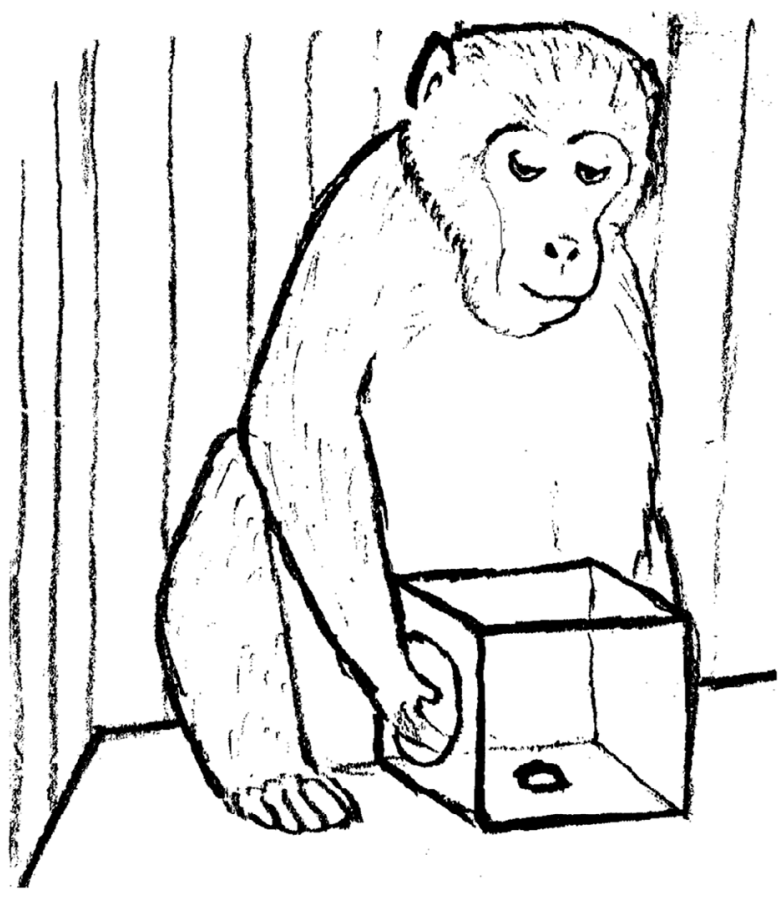

Fig. 2. Monkey's work in the operant task consisting of extracting reinforcement from a transparent hollow cube.

sponses reached $90 \%$, the colored background was removed, allowing final transfer to categorization using images alone. The animals' work was optimized by decreasing the acceptable time interval during which the animal could respond to the stimuli, which ultimately led to a decrease in the latency of the monkeys' responses.

At the final stage, the task was supplemented by addition of new images of other butterflies or faces.

Food reinforcement extraction task. Studies of the perception of spatial relationships in the monkeys' personal space used an operant task with accessing a reinforcement in the spatial complexity of the context of the environment. The experimental apparatus was a cage in which the monkey was given a transparent hollow cube with sides of length $15 \mathrm{~cm}$. One wall of the cube had an opening $12 \mathrm{~cm}$ in diameter. The cube could be fixed in front of the monkey in five different positions: with the opening upward, facing the animal, at right, at left, and away from the animal (Fig. 2). The food reinforcement was loaded into the center of the cube - pieces of tasty food (dried fruit, candied fruit, etc.) weighing 2-3 g. The experiment was performed in the presence of the experimenter, either visually noting the features of the monkey's behavior and determining the experimental parameters or making video recordings of the animal performing the task for subsequent analysis.

The monkey was initially trained to work in the apparatus. The cube, in one of its positions, with the reinforcement within it, was moved into the experimental cage. The duration of a single presentation of the cube was limited to
3 min - enough time for a trained and motivated animal to solve the task. The position of the opening in the cube was changed in pseudorandom order for each subsequent trial, on the one hand to avoid presenting the task in the same orientation several times in a row and on the other to ensure that all opening positions were presented in the experiment in the same proportions $(1 / 5=20 \%)$.

The adaptation period could last several experimental days depending on the individual features of the monkeys, including their activity and the intensity of their exploratory behavior. The monkey could solve the task at any moment, generally by reaching the reinforcement in one of the cube opening positions easy for reaching the reinforcement: above or facing the animal. The animal then started to solve the task successfully with these opening positions and to learn solutions with the opening in more difficult positions. The greatest difficulty could arise in solving the task with the opening position away from the animal.

After animals had learned to solve the spatial task with any opening position, the experiment started to record the animals' behavioral indicators during task solution, especially its actions with the cube. All manipulatory actions by the monkeys with the cube could be discriminated in terms of three groups: 1) correct actions - the monkey solved the task and took the reinforcement through the opening in the cube using the hand; 2) erroneous actions - the monkey tried to take the reinforcement through the solid wall of the cube: the action was appropriate to the task but incorrect for the position of the opening; and 3) other actions - attempts to break, gnaw, or shake the cube, i.e., actions which would not lead to task solution with the opening in any position.

With a good level of motivation, the animals could successfully solve about 50 task trials in an experimental day. The animal's motivation then decreased, which led to nonuniformity in the results obtained.

Visual observations of the monkeys' behavior during the experiment determined the task solution time (using a stopwatch), the presence or absence of erroneous actions during task solution and the hand used by the monkey to take the reinforcement in solving the task. Video recordings of experiments allowed later examination to determine the time taken, the wall through which the animal tried to perform the action, and the hand used for the action for each erroneous action; the total numbers of these errors per task presentation were also determined.

Results. Learning dynamics of monkeys in an oculomotor task. The success of training monkeys in the conditions of an operant task depends largely on the animal's level of motivation. The reinforcement used here was fruit juice, generating the need to control the quantity of liquid needed. The level of deprivation should not affect the overall physiological state of the animal. The animals' state was monitored by weighing twice a week. Weight change data are presented in Fig. 3. The experiments were performed over a period of about 200 days. The first weighing was on 


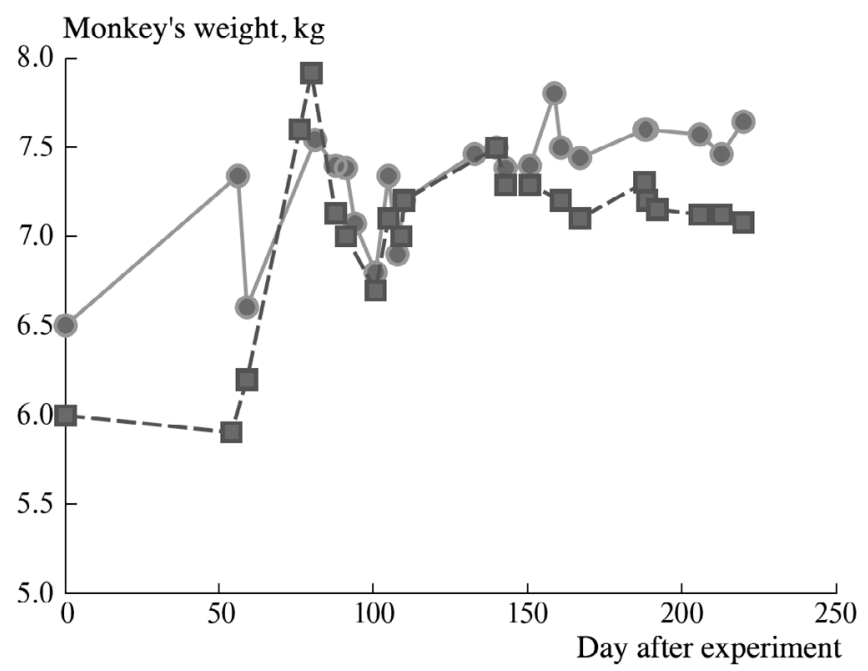

Fig. 3. Changes in animals' body weight during the whole experiment with training to an oculomotor task. Circles - monkey VL; squares - monkey GR.

the day when the animals arrived in the animal house. These results show that weight stabilized over the experiment period, with some tendency to increase. This is associated with the fact that we did not transfer the animals to dry feed during training. The daily diet consisted of cereal porridge combined with small quantities of fruits and vegetables. The standard amount of liquid was at least $150 \mathrm{ml}$, which the animal generally received during training. Thus, involvement in our experiments had no effect on the animals' general state. We were able to select keeping conditions which promoted progress in training without affecting the animals' wellbeing.

Summary data on training monkeys to cognitive tasks are presented in Fig. 4. Before training to the main task, the animals were acclimated to the primatology chair and use of the levers. The initial segment of the plot shows that the animals had already formed a primary association between the appearance of the stimulus and the need to move the lever to obtain the reinforcement. At this state, the visual stimulus occupied the whole of the screen. Thus, in the simplest conditions, "Fix" (just one square appeared at the center of the screen), animals VL and GR demonstrated high levels of correct responses. After stabilization of the skill, we transferred to acquisition of a generalized response to the visual stimulus. This was done by gradually altering the size of the square located at the center of the screen. The moments at which the paradigm changed are shown by vertical lines (Fig. 4, $a, c$ ). We see that the animals quite quickly assimilated the task, such that they could quickly progress to the next set of conditions. The critical moment was formation of the association between the change in visual stimulus brightness and the need to respond to this event (Fig. 4, $a, c$; vertical line B). This change in experimental conditions, as expected, led to an increase in the number of errors. Fixation of the response to the decrease in stimulus brightness took
932 trials in monkey GR, over five experimental days, while animal VL learned the need to respond to the change in brightness over 3165 trials taking eight experimental days.

Clearly, the most critical moment in learning was the stage with addition of the TrS. To understand the rule, the animal required some number of training days in order to reach a high level of correct reactions. The second critical moment was the appearance of heterogeneous PrS at stage "H" (Fig. 4, $a, c$ ). In this case, the animal had reached the $60 \%$ level of correct reactions for three experimental days. Further changes in the experimental conditions, with addition of a large number of stimuli and a reduction in stimulus size, had only minor effects on the proportion of correct reactions. As a rule, the level decreased to $70 \%$. Along with the increase in the proportion of correct reactions, there was a simultaneous decrease in their latent periods (Fig. 4, $b, d$ ), which provided added confirmation of fixation of the skill.

Training to the complex images categorization task. The observation period for the image categorization task was about 600 days. The training process could be divided into several stages, preliminary training of the animals took 250-285 days. By the end of this period, monkey BL could manipulate the joystick selecting one of the two directions of movement depending on the colored square presented in the upper or lower part of the screen. At this stage, the proportion of correct reactions was about $90 \%$. A drop in the proportion of correct reactions to the random level was linked with the change in paradigm, in which stimuli were presented at the center of the screen (Fig. 5, vertical line "A"). An analogous decrease in the proportion of correct responses occurred with new modifications to the task: use of an image as stimulus and shortening of the time interval allotted for the response (vertical lines B and C, respectively, Fig. 5, $a$ ). The proportion of correct responses by experimental days 4-8 reached about $80 \%$. 

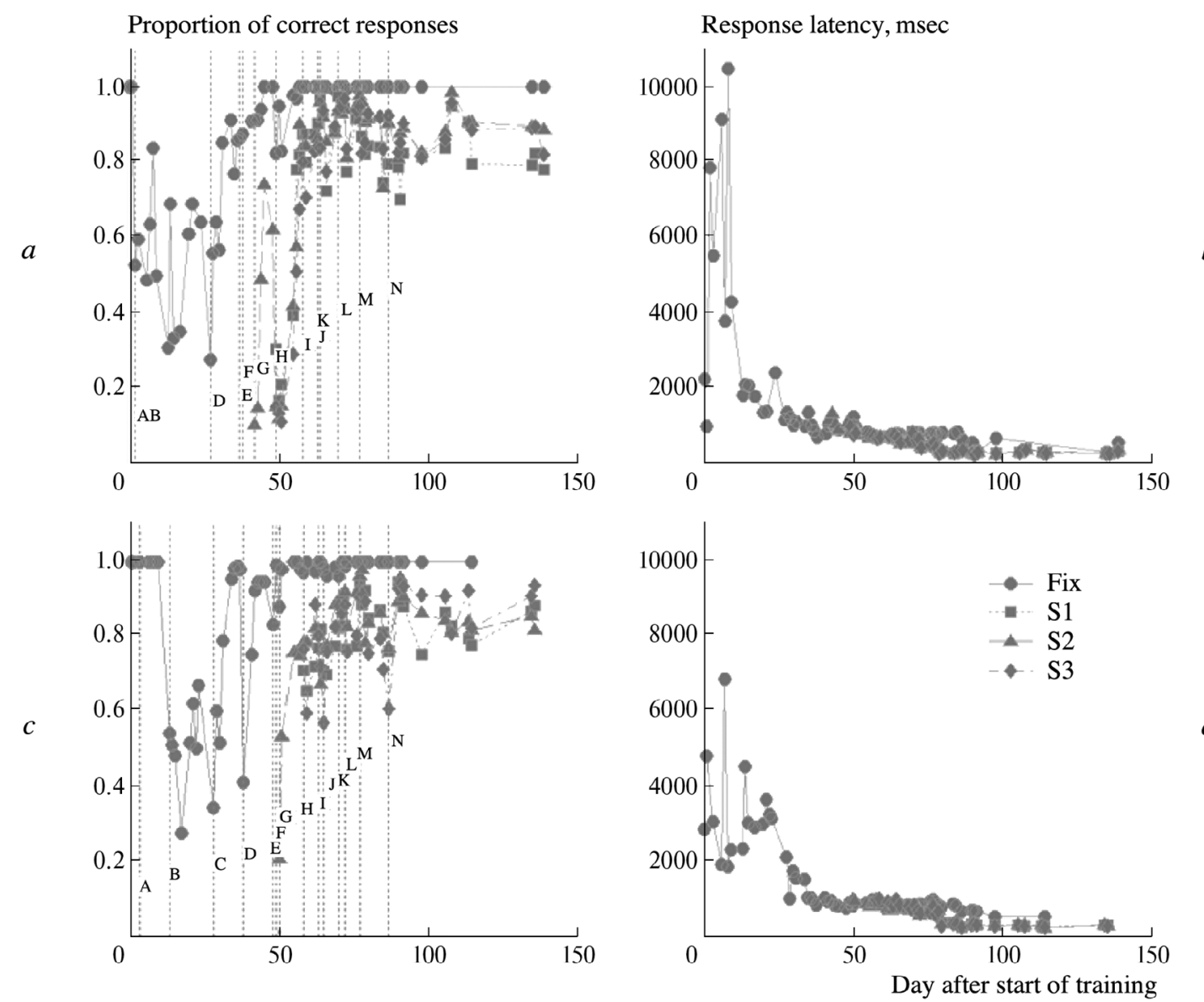

Fig. 4. Changes in the proportion of correct responses an latencies in the oculomotor task over time. $a, c$ ) Proportions of correct responses by monkeys VL and GR, respectively. Vertical lines designate the moments at which the experimental conditions changed. A) Stimulus size $21.1^{\circ} \times 21.1^{\circ}$; B) addition of a reduction in the intensity of the $\mathrm{TrS}$; C) change in size of CS to $18.5^{\circ}$; D) CS size $15.9^{\circ}$; E) CS size $13.3^{\circ}$; F) CS size $10.7^{\circ}$; G) addition of PrS without delay into the regime, with stimulus size $8^{\circ} ; \mathrm{H}$ ) addition of PrS to regime with delay and overlap; I) sizes of stimuli $2.1^{\circ}$; J) sizes of stimuli $1.1^{\circ}$, stimuli now appearing over the whole plane of the screen; K) stimulus size $0.5^{\circ}$, decreased in proportion of central stimuli; L) stimulus size $0.3^{\circ} ; \mathrm{M}$ ) stimulus size $0.2^{\circ} ; \mathrm{N}$ ) decreased in difference between $\operatorname{PrS}$ and $\operatorname{TrS}$ brightness. $b, d$ ) Changes in latencies of reactions of monkeys VL and GR, respectively.

Fixation of the motor task is illustrated in panels $b-e$ of Fig. 5. Movements were initially inaccurate and suboptimal and the animals moved the joystick through quite complex trajectories (Fig. 5, $b, c$ ). During the learning process, the trajectory became shorter and closer to the optimal (Fig. $5, d, e$ ). It should be noted that decreases in trajectory length occurred after reductions in the time allowed for the reaction. As soon as the allotted reaction time was too short for a long movement to be executed, the animal optimized the limb movement. Mean reaction time decreased during fixation of the motor skill (Fig. 5, $f-i$ ).

The distribution of the reaction times for correct responses to stimuli in the categorization task was bimodal (Fig. 5, $f-i$ ), which we link with the different nature of the operant reactions. Movement of the joystick in the "away" direction had shorter latency than movements in the "towards" direction. As "away" movements in response to images of butterflies were added to the task earlier than "towards" movements, they were more fixed and, as a result, more optimal. We planned to record neuron activity in animals during execution of this task, so it was important to equalize responses to different groups of stimuli as much as possible, gradually decreasing the time allotted to execution of the operant reactions. The reaction times for "towards" and "away" movements decreased significantly, though we were unable to equalize them completely during the experiments. Reaction times for "towards" movements underwent stronger changes as the latent period of the "towards" reaction was initially greater.

Training of monkeys to the bait extraction task. Motor activity and the individual features of motor behavior could be assessed using data on mean task solution times and the active hand both on average and for each opening position individually. The features of the execution of erroneous actions can be used for assessing the perception by animals of the cognitive function of spatial relationships of objects in 

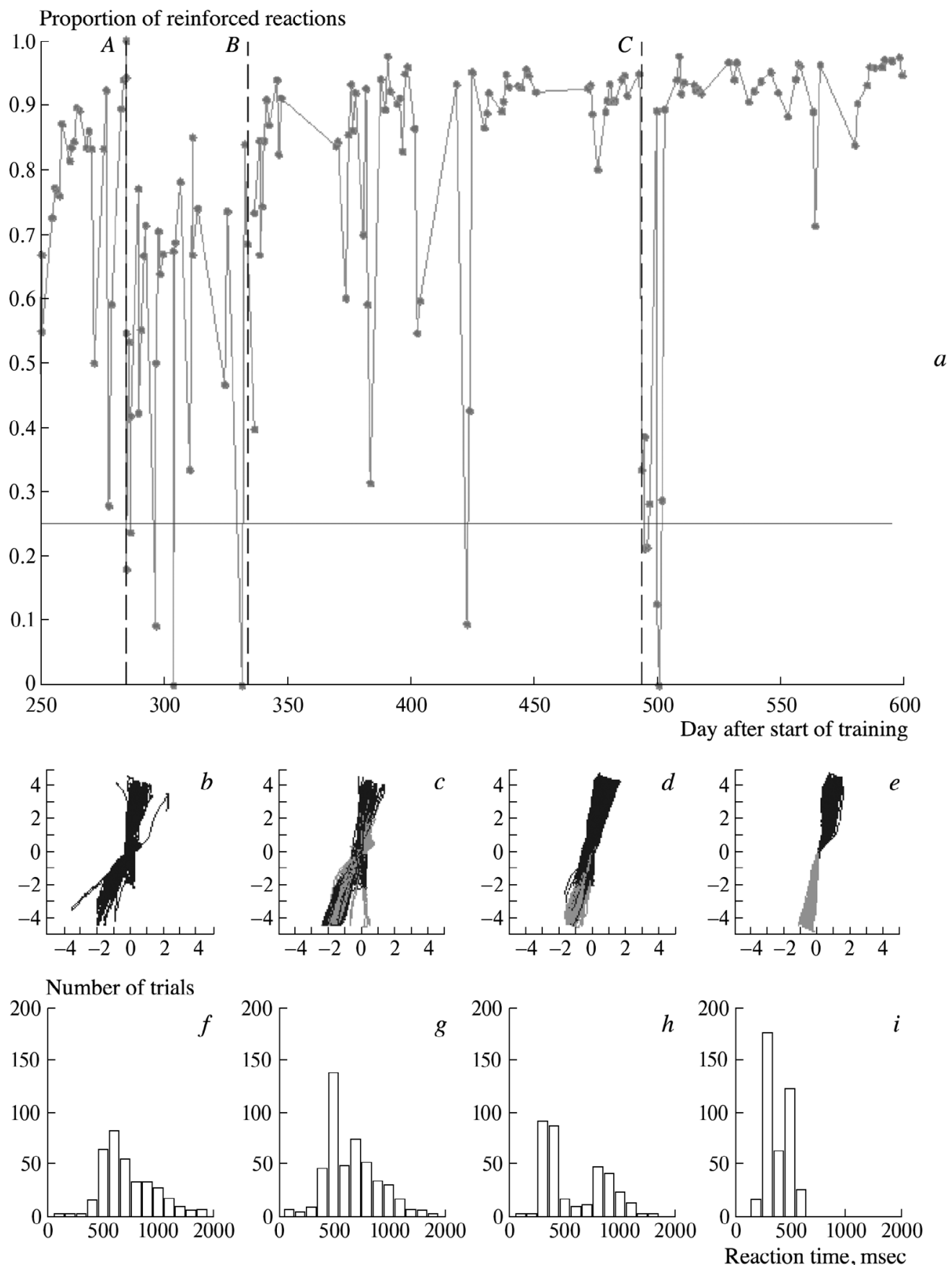

Fig. 5. Dynamics of behavioral parameters during training of monkey BL to the complex visual stimulus categorization task. a) Dynamics of the proportion of correct responses in visual stimulus categorization experiments. The horizontal continuous line shows the probability of performance of the two-stimulus categorization task at the random level (0.25). Vertical dotted lines show the moments at which the task was modified: $A$ ) blue and yellow squares presented at the center of the screen and not above and below, as previously; $B$ ) color of squares remains as the background for images of a face or butterfly; $C$ ) start of gradual reduction in reaction time from $1500 \mathrm{msec}$ to $550 \mathrm{msec} . b-e$ ) joystick movement trajectories on days 38, 293, 475, and 594 after training started. Joystick movement trajectories in the "away" direction are shown in black (in response to the butterfly image); "towards" movements are shown in gray (to the face image). $f-i$ ) Distributions of reaction times on the days indicated after training started.

near (personal) space. The dynamics of changes in measures allow the learning ability of the animals to be evaluated.

Figure $6, a, b$ shows typical measures of the animals'; work in the experiment. Curve (1) corresponds to the proportion of successfully solved task presentations by experi- mental day. This clearly shows that after three days of adaptation, the monkey completely learned to solve the task over two days. The proportions of error-free solutions of the task by the monkey (solution of the task on the first attempt to take the reinforcement), along with mean reaction times on 

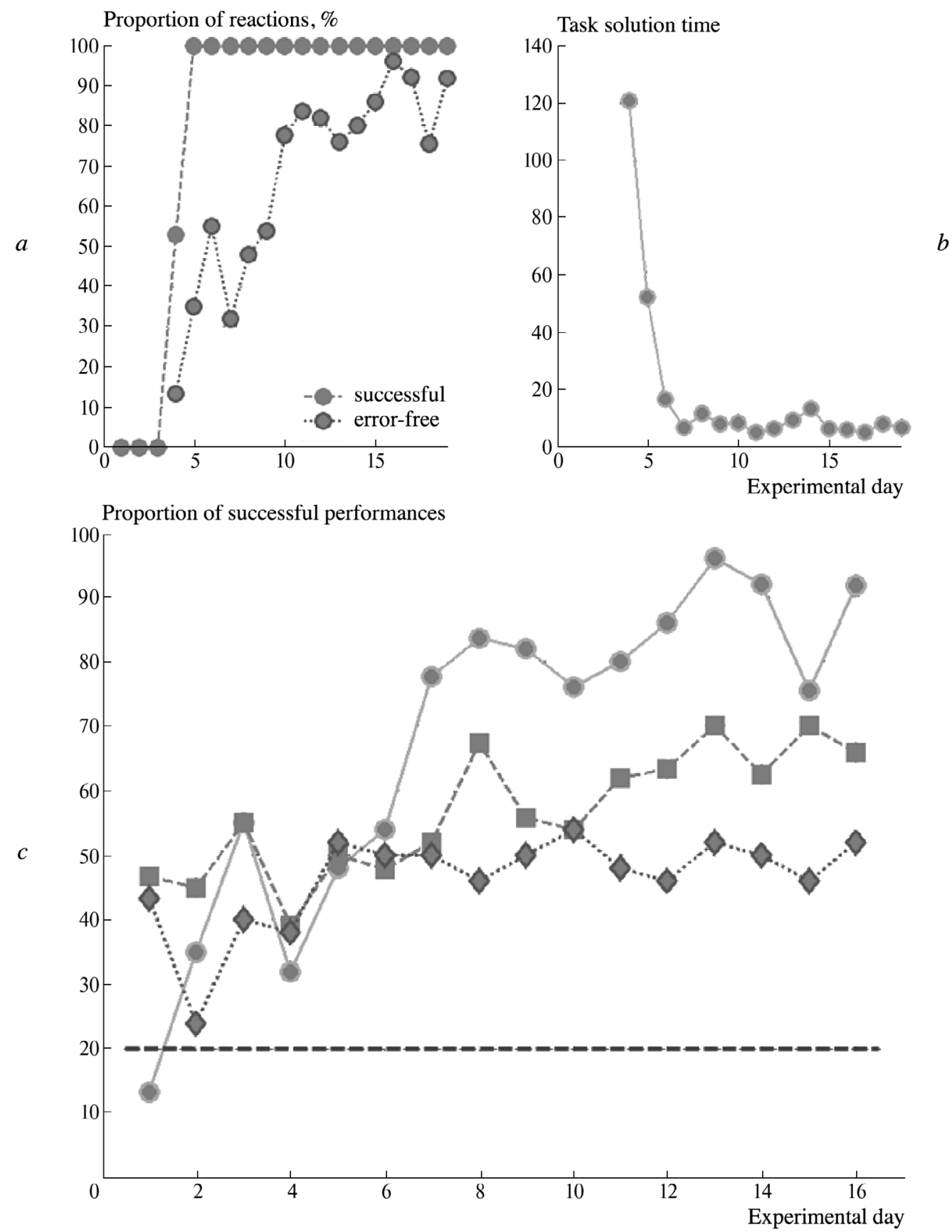

Fig. 6. Training of monkeys to the task consisting of extracting a food reinforcement from a hollow cube. a) Mean proportion of performances for three animals on successful solution of the task on different experimental days; $b$ ) mean task solution time for three animals depending on the number of the experimental day; $c$ ) proportion of task solutions for monkeys VL $(\bullet)$, GR $(\bullet)$, and DK $(\bullet)(\%)$. Horizontal dotted lines show the probability of random task performance $(1 / 5=20 \%)$. The abscissa shows experimental days.

the experimental day, are shown from the moment at which solution of the spatial task by the monkey started. The dynamics of task solution time clearly show rapid formation of the set of actions required for successful task solution.

The dynamics of the proportion of error-free task solutions for three monkeys are shown in Fig. 6, $c$. This shows that monkeys learned to solve the task: the proportion of error-free solutions was notably and significantly greater than the corresponding random task solution level (about one in five of all task presentations would be solved correctly by working randomly). Attention is drawn to the different proportions of error-free task solutions reached in the experiment, the rate of learning to achievement of this level, and the stability of the proportion of error-free solutions between experimental days for each monkey. 
Discussion. This report presents standardized protocols for training monkeys to complex cognitive tasks. Assessment of the cognitive capacity described here is used in models of central nervous system pathology [Weed et al., 2004], studies of drug actions [Schneider et al., 2015; Soto et al., 2013a, 2013b], studies of the function of individual brain structures [Forcelli et al., 2014], and descriptions of normal aging [Didier et al., 2016; Kohama et al., 2016; Nagahara et al., 2010; Zeamer et al., 2012]. Standard behavioral methods must be used for the results of different experiments to be compared. It is important to define the parameters characterizing the monkeys' behavior and the course of the learning process. Acquisition of the oculomotor task provides the opportunity for simultaneous assessment of several parameters characterizing the functional state of the brain in primates. Our approach allows assessment of both the motor components of the animals' response and the cognitive components. The motor components can be assessed after full assimilation of the task by the animal on the basis of recording hand movements, saccadic eye movements, and coordination between hand and eye movements. Cognitive characteristics can be assessed using variations in delayed-response task which require retention of working or long-term memory. Testing in the oculomotor task proposes use of a drink reinforcement. The drink motivation does not "compete" with the food motivation, such that tasks based of extraction of bait from a transparent cube with a single opening can be run in parallel. Within a single experimental day, different characterizations of the animals' cognitive capacities can be obtained, which is very important for assessment of the dynamics of recovery of cognitive functions after any kind of action (for example, drug administration, extirpation of brain structures, the actions of ionizing radiation, etc.). We have shown that regardless of the task (oculomotor or categorization), the animals were able to assimilate the "rules" determining receipt of the reward quickly. The total duration of training in the oculomotor task was of the order of three months, after which the skill stabilized.

It is important to note that the animals' ability to carry out complex behavioral tasks on testing of cognitive functions depends on the individual's age. Furthermore, the cognitive test battery is often used in model experiments on aging [Didier et al., 2016; Kohama et al., 2016; Nagahara et al., 2010; Zeamer et al., 2012]. The results of the present study do not allow us to run a detailed assessment of the data in relation to the animals' age because of the small number of animals and the large differences in their ages. Nonetheless, readers' attention should be drawn to the need to take account of the monkeys' ages in assessing their cognitive capacities. Furthermore, other individual features - the course of early ontogeny, traumatizing experience, the details of their keeping - should always be considered in analyzing behavioral data reflecting the features of cognitive activity in primates.

The tendency of the world community to increase the wellbeing of animals used in scientific experiments has in- creased in recent years. This tendency includes the training of animals and is apparent as new approaches to decrease animals' discomfort and to approach training not by deprivation but by interesting the animal in the task. One such system was developed at Göttingen [Berger et al., 2017]. The animals were trained without leaving their cages and could be engaged in their task at any time. The pluses of this approach are obvious: the animals do not experience stress due to being moved from their home cages to the experimental context; it also provides the opportunity to carry out tasks when they are most active. This naturally raises the question of the level of motivation and the authors demonstrated that monkeys were involved in performing the task for at least several months, even when they had free access to water and food. Other authors have suggested similar approaches for experimental testing of animals and selecting the most appropriate approaches for particular behavioral tasks [Calapai et al., 2017; Tulip et al., 2017].

Testing of visual function in primates becomes particularly relevant in the light in the introduction of new approaches to the treatment of degenerative diseases of the retina using optogenetic technologies [Firsov, 2017].

This study was supported financially by the Russian Foundation for Basic Research (Project No. 17-29-01027 “ofi_m").

\section{REFERENCES}

Asaad, W. F., Santhanam, N., McClellan, S., and Freedman, D. J., "Highperformance execution of psychophysical tasks with complex visual stimuli in MATLAB," J. Neurophysiol., 109, No. 1, 249-260 (2013).

Berger, M., Calapai, A., Stephan, V., et al., "Standardized automated training of rhesus monkeys for neuroscience research in their housing environment," J. Neurophysiol. (Epub ahead of print).

Brainard, D. H., "The psychophysics toolbox," Spat. Vis. 10, 433-436 (1997).

Calapai, A., Berger, M., Niessing, M., et al., "A cage-based training, cognitive testing and enrichment system optimized for rhesus macaques in neuroscience research," Behav. Res. Methods, 49, No. 1, 35-45 (2017).

Camus, S., Ko, W. K., Pioli, E., and Bezard, E., "Why bother using non-human primate models of cognitive disorders in translational research? Neurobiol," Learn. Mem., 124, 123-129 (2015).

Capitanio, J. P. and Emborg, M. E., "Contributions of non-human primates to neuroscience research,” Lancet, 371, No. 9618, 1126-1135 (2008).

Chelonis, J. J., Cox, A. R., Karr, M. J., et al., "Comparison of delayed matching-to-sample performance in monkeys and children," Behav. Processes, 103, 261-268 (2014).

Chiueh, C. C., Markey, S. D., Burns, R. S., et al., "Neurochemical and behavioral effects of MPTP in rat, guinea pig and monkey," Psychopharmacol. Bull., 20, 548-553 (1984).

DeLong, M. R., "Primate models of movement disorders of basal ganglia origin," Trends Neurosci., 13, 281-285 (1990).

Didier, E. S., MacLean, A. G., Mohan, M., et al., "Contributions of nonhuman primates to research on aging," Vet. Pathol., 53, No. 2, 277-290 (2016).

Firsov, M. L., "Perspectives for optogenetic prosthetization of the retina," Zh. Vyssh. Nerv. Deyat., 67, No. 5, 53-62 (2017).

Forcelli, P. A., Palchik, G., Leath, T., et al., "Memory loss in a nonnavigational spatial task after hippocampal inactivation in monkeys," Proc. Natl. Acad. Sci. USA, 111, No. 11, 4315-4320 (2014). 
Goldman-Rakic, P. S., "Cellular basis of working memory," Neuron, 14, 477-485 (1995).

Hanbury, D. B., Peiffer, A. M., Dugan, G., et al., "Long-term cognitive functioning in single-dose total-body gamma-irradiated rhesus monkeys (Macaca mulatta)," Radiat. Res., 186, No. 5, 447-454 (2016).

Jenner, P., "From the MPTP-treated primate to the treatment of motor complications in Parkinson's disease," Parkinsonism Relat. Disord., Suppl. 4, 18-23 (2009).

Kleiner, M., Brainard, D., and Pelli, D., "What's new in Psychtoolbox-3?" Perception, ECVP Abstr, Suppl. (2007).

Kohama, S. G., Renner, L., Landauer, N., et al., "Effect of ovarian hormone therapy on cognition in the aged female rhesus macaque," J.Neurosci., 36, No. 40, 10416-10424 (2016).

Langston, J. W., Forno, L. S., Rebert, C. S., and Irwin, I., "Selective nigral toxicity after systemic administration of 1-methyl-4-phenyl 1-1,2,3,6, tetrahydropyridine (MPTP) in the squirrel monkey," Brain Res., 292, 390-394 (1984).

Latanov, A. V., Tereshchenko, L. V., Yudin, A. G., and Shul'govskii, V. V., "Residual impairments to operant behavior in monkeys after recovery form MPTP-induced parkinsonism-like syndrome," Ros. Fiziol. Zh., 83, No. 12, 159-162 (1997).

Lind, J., Enquist, M., and Ghirlanda, S., "Animal memory: A review of delayed matching-to-sample data," Behav. Processes, 117, 52-58 (2015).

McMahon, D. B., Russ, B. E., Elnaiem, H. D., et al., "Single-unit activity during natural vision: diversity, consistency, and spatial sensitivity among AF face patch neurons," J. Neurosci., 35, No. 14, 5537-5548 (2015).

Moss, M. B., Killiany, R. J., Lai, Z. C., et al., "Recognition memory span in rhesus monkeys of advanced age," Neurobiol. Aging, 18, 13-19 (1997).

Nagahara, A. H., Bernot, T., and Tuszynski, M. H., "Age-related cognitive deficits in rhesus monkeys mirror human deficits on an automated test battery," Neurobiol. Aging, 31, No. 6, 1020-1031 (2010).

Pelli, D. G., "The VideoToolbox software for visual psychophysics: Transforming numbers into movies," Spat. Vis., 10, 437-442 (1997).

Phillips, K. A., Bales, K. L., Capitanio, J. P., et al., "Why primate models matter," Am. J. Primatol., 76, No. 9, 801-827 (2014).

Porras, G., Li, Q., and Bezard, E., "Modeling Parkinson's disease in primates: The MPTP model," Cold Spring Harb. Perspect. Med., 2, a009308 (2012).

Rizzolatti, G., Fadiga, L., Gallese, V., and Fogassi, L., "Premotor cortex and the recognition of motor actions," Brain Res. Cogn. Brain Res., 3 , No. 2, 131-141 (1996).

Rodriguez, J. S. and Paule, M. G., "Working memory delayed response tasks in monkeys," in: Methods of Behavior Analysis in Neuroscience, Buccafusco, J. J. (ed.), C Press/Taylor \& Francis, Boca Raton (2009), 2nd ed., https://www.ncbi.nlm.nih.gov/books/NBK5227/.

Roelfsema, P. R. and Treue, S., "Basic neuroscience research with nonhuman primates: a small but indispensable component of biomedical research," Neuron, 82, No. 6, 1200-1204 (2014).
Schneider, J. S., Williams, C., Ault, M., and Guilarte, T. R., "Effects of chronic manganese exposure on attention and working memory in non-human primates," Neurotoxicology, 217-222 (2015).

Soto, P. L., Ator, N. A., Rallapalli, S. K., et al., "Allosteric modulation of GABA(A) receptor subtypes: effects on visual recognition and visuospatial working memory in rhesus monkeys [corrected]," Neuropsychopharmacology, 38, No. 11, 2315-2325 (2013a).

Soto, P. L., Dallery, J., Ator, N. A., and Katz, B. R., "A critical examination of best dose analysis for determining cognitive-enhancing potential of drugs: studies with rhesus monkeys and computer simulations," Psychopharmacology (Berlin), 228, No. 4, 611-622 (2013b).

Taffe, M. A. and Taffe, W. J., "Rhesus monkeys employ a procedural strategy to reduce working memory load in a self-ordered spatial search task," Brain Res., 1413, 43-50 (2011).

Tereshchenko, L. V., Anisimov, V. T., Shul'govsky, V. V., and Latanov, A. V., "Early changes of saccade parameters in monkeys at the developments of MPTP-induced hemiparkinsonism," Perception, 44, No. 8-9, 1054-1063 (2015).

Tereshchenko, L. V., Molchanov, S. A., Kolesnikova, O. V., and Latanov, A. V., "Functional asymmetry of hand movements in monkeys (Macaca mulatta)," Zh. Vyssh. Nerv. Deyat., 62, No. 4, 431-439 (2012).

Tereshchenko, L. V., Yudin, A. G., Kuznetsov, Yu. B., et al., "Impairments to saccadic eye movements on development of MPTP-induced syndrome in monkeys," Byull. Eksperim. Biol. Med., 133, No. 2, 215218 (2002).

Tsao, D. Y., Moeller, S., and Freiwald, W. A., "Comparing face patch systems in macaques and humans," Proc. Natl. Acad. Sci. USA, 105, No. 49, 19514-19519 (2008)

Tulip, J., Zimmermann, J. B., Farningham, D., and Jackson, A., “An automated system for positive reinforcement training of group-housed macaque monkeys at breeding and research facilities," J. Neurosci. Meth., 285, 6-18 (2017).

Weed, M. R., Gold, L. H., Polis, I., et al., "Impaired performance on a rhesus monkey neuropsychological testing battery following simian immunodeficiency virus infection," AIDS Res. Hum. Retroviruses, 20, No. 1, 77-89 (2004).

Wichmann, T. and DeLong, M. R., "Pathophysiology of Parkinson's disease: the MPTP primate model of the human disorder," Ann. NY Acad. Sci., 991, 199-213 (2003).

Williams, K. C., Corey, S., Westmoreland, S. V., et al., "Perivascular macrophages are the primary cell type productively infected by simian immunodeficiency virus in the brains of macaques: implications for the neuropathogenesis of AIDS," J. Exp.Med., 193, No. 8, 905-915 (2001).

Yan, T., Rizak, J. D., Yang, S., et al., “Acute morphine treatments alleviate tremor in 1-methyl-4-phenyl-1,2,3,6-tetrahydropyridine-treated monkeys," PLoS One, 9, No. 2, e88404 (2014)

Zeamer, A., Clark, K., Bouquio, C., et al., "Impaired spatial working memory learning and performance in normal aged rhesus monkeys," Behav. Brain Res., 232, No. 1, 287-293 (2012). 